an IBS symptom questionnaire, the Hospital Anxiety and Depression Scores (HAD), and WHO-QoL questionnaire. The Primary outcome was a reduction in total cost over the follow up period. Secondary outcomes were a reduction in HADS and an increase in WHO-QOI

Results 24 patients have been enrolled into the clinic to date. The first 5 patients have been analysed. There were 22 fewer contacts after COGS attendance (inpatient, outpatient, emergency department, and day case). Follow up was between 5 and 7 months. This was a reduction of 4.4 contacts per patient (0.6 - 0.9 contacts per patient per month) Overall costs were $£ 8,392$ lower, equivalent to $£ 1,678$ saving per patient ( $£ 240-£ 336$ per patient per month). Individual WHO-QOL-BREF results have shown up to a 23 point increase. Staff reflections can be summarised as a growing confidence in the power of listening and acceptance of incremental gains in symptoms.

Conclusions We have demonstrated that a novel multidisciplinary approach to patients in gastroenterology is cost effective, leads to increased staff satisfaction and have preliminary data showing improved patient reported outcomes.

\section{PWE-081 HYDROGEN BREATH TESTING: HIGH INCIDENCE OF SMALL INTESTINAL BACTERIAL OVERGROWTH DIAGNOSIS USING LACTULOSE VERSUS GLUCOSE}

Elisabeth Kirton*, Lois Anderson-Leary, John Gallagher, Samantha Hewitt, Jennifer Burke, Warren Jackson. Hull and East Yorkshire Hospitals NHS Trust, Castle Hill Hospital, UK

\subsection{6/gutjnl-2019-BSGAbstracts.401}

Background A hydrogen breath test is an inexpensive, noninvasive and safe diagnostic test used to investigate intestinal disorders, including suspected Small Intestinal Bacterial Overgrowth (SIBO). The recent publication of the North American Consensus statement ${ }^{1}$ suggests a rise $\geq 20$ ppm of hydrogen within $90 \mathrm{~min}$ as an ideal threshold for a positive test to suggest the presence of SIBO following ingestion of glucose or lactulose. As lactulose is a non-digestible disaccharide, it is possible that such a rise during a lactulose test is due to fermentation in the colon, rather than an indicator of SIBO. A small retrospective audit was undertaken to investigate how many patients with a positive lactulose test for SIBO had a subsequent positive glucose test.

Methods Adult patients who had previously attended for a lactulose hydrogen breath test which established the presence of hydrogen-producing bacteria and met the diagnostic criteria for SIBO were included in this audit. All patients attended for a glucose hydrogen breath test within 6 weeks of their lactulose breath test, and had not received antibiotic therapy during this time period. After a baseline reading, patients provided a breath-hydrogen sample every $15 \mathrm{~min}$ for $60 \mathrm{~min}$ post ingestion of a glucose solution. Further breath hydrogen samples were then taken every $30 \mathrm{~min}$ for $180 \mathrm{~min}$. Results were analysed to determine the number of patients who met the diagnostic criteria for SIBO $(\geq 20 \mathrm{ppm}$ rise in hydrogen above baseline within $180 \mathrm{~min}$ ).

Results 18 patients $(11 \mathrm{~F}, 7 \mathrm{M})$ were included in this small audit. 2 patients (F) were found to be positive for SIBO, whereas 16 patients $(9 \mathrm{~F}, 7 \mathrm{M})$ were concluded to be negative, as they did not demonstrate a significant rise in expired hydrogen ( $\geq 20$ ppm above baseline) within $180 \mathrm{~min} .11 .1 \%$ of patients included in this audit had SIBO confirmed with a glucose hydrogen breath test, despite all having a positive lactulose test using the criteria suggested in the North American Consensus paper.

Conclusion Based on the lactulose results alone, the majority of patients in this audit (88.9\%) would have been reported as having a SIBO, despite a negative glucose test. Using lactulose alone as a substrate to diagnose SIBO may therefore provide a false positive result, potentially leading to misdiagnosis and inappropriate use of antibiotics.

\section{REFERENCE}

1. Rezaie A, et al. Hydrogen and Methane-Based Breath Testing in Gastrointestinal Disorders: The North American Consensus. The American journal of gastroenterology. 2017; 112(5):77-4.

\section{PWE-082 THE ART OF COMBINING CLINICAL INTUITION AND NUCLEAR MEDICINE IN DIAGNOSING BILE ACID MALABSORPTION}

James Lok*, Mike Peters, Bilal Mateen, Robert Logan. King's College Hospital, London, UK

\subsection{6/gutjnl-2019-BSGAbstracts.402}

Introduction NICE DG7 (2012) highlights the problems of SeHCAT scanning in the diagnosis of Bile Acid Malabsorption (BAM) for patients presenting with IBS-D. 2018 BSG guidelines for the investigation of chronic diarrhoea recommend both SeHCAT scanning (for the diagnosis of BAM) and colonoscopy (for diagnosis of microscopic colitis), which can occur in up to $25 \%$ and $10 \%$ of cases of IBS-D, respectively. Nocturnal symptoms and incontinence are 'hallmark' symptoms for microscopic colitis. This retrospective study evaluates the use of SeHCAT scanning at a London teaching hospital and explores if similar 'hallmarks' exist for BAM.

Methods The patient cohort was identified by searching the CRIS database from inception to Oct 2018 whilst clinical data was extracted from the electronic patient record system. SeHCAT scans were performed using ${ }^{75} \mathrm{Se}$-hemoefolic acid taurine capsules at a dose of $0.4 \mathrm{MBq}$ and $0.3 \mathrm{mSv}$. Gamma counts were recorded 3 hours and then 7 days post capsule administration using a camera detecting the $27-80 \mathrm{keV}$ photo peak and retentions of $<15 \%$ were considered abnormal. Data collected included demographics, clinical presentation (abdominal pain, diarrhoea, bloating, incontinence, urgency, nocturnal symptoms) and relevant past medical history. Multivariate analysis of symptoms was performed using a generalised linear model with elastic net and $\mathrm{K}$ fold cross validation. Patients with terminal ileal resections or IBD were excluded from subsequent analysis.

Results From Jan 2013 to Oct 2018, 524 patients underwent a SeHCAT scan (65\% female, mean age 51) and the rate of testing increased sequentially from 26 per annum to 124 per annum over five years. Detailed statistical analysis of symptom cluster failed to identify any 'hallmark', but nevertheless $49.8 \%$ of patients had abnormal tests, $56.7 \%$ of which were requested by $4 / 16$ consultant gastroenterologists. Sixty-four patients developed diarrhoea following a laparoscopic cholecystectomy $(n=2476)$ of whom $41(64 \%)$ had positive scans. 
In the same time period as this study, 1719 patients underwent colonoscopy and biopsy due to unexplained diarrhoea.

Conclusions These data confirm that BAM is common but is under-investigated in comparison with microscopic colitis. Although it is not associated with any 'hallmark' symptoms, it is readily identified by experienced clinicians as suggested by the high pick up rate, presumably on the basis of clinical acumen. It occurs in approximately $2 \%$ of patients following cholecystectomy, although the true prevalence in this population may be greater. These data also support the BSG guideline audit recommendation of SeHCAT in IBS-D as a quality improvement target.

\section{PWE-083 PREVALENCE OF OPIOID THERAPY IN PATIENTS WITH FUNCTIONAL GASTROINTESTINAL DISORDERS REVIEWED AT A TERTIARY CENTRE}

${ }^{1,2}$ Amir Mari*, ${ }^{2}$ Marcella Pesce, ${ }^{2}$ Kalp Patel, ${ }^{2}$ Fotios Lezgidis, ${ }^{2}$ Anton Emmanuel, Natalia Zarate-Lopez. ${ }^{1}$ Nazareth Hospital Emms Trust, Nazareth, Israel; ${ }^{2}$ Gl Physiology Unit, UCLH, London, UK

\subsection{6/gutjnl-2019-BSGAbstracts.403}

Introduction The appropriateness of chronic opioid use in patients with non-cancer pain is increasingly challenged due to limited evidence of its efficacy and the risk of problematic use and dependence. The side effects of opioids on gut function suggest this therapy should be particularly avoided in patients with functional gastrointestinal (GI) disorders. Data on the prevalence of opioid use in patients with functional GI disorders is unknown. We aimed to evaluate the prevalence of opioid use in functional GI patients referred to a tertiary Neurogastroenterology clinic and to determine the risk factors for their initiation.

Methods 100 consecutive outpatients (85 females, 37 \pm 15 years) with functional GI disorders referred for a specialist opinion at our tertiary referral centre were retrospectively reviewed. Two independent researchers (AM and FL) carried out a detailed chart evaluation of the presence and type of comorbidities and concomitant pharmacotherapy. Patients were also specifically asked about opioid use, the duration of therapy and the clinical indication for starting the opioids.

Results Twenty-one patients (20F, 38 \pm 12 years) were taking chronic opioid therapy. The main reason for opioid initiation was chronic musculoskeletal pain (12 patients, 57\%), chronic abdominal pain (5 patients, 24\%) and chronic pelvic pain (1, $5 \%)$. The mean duration of the opioid treatment was $2.7 \pm 1$ years. The risk factors associated with opioids use are depicted in table 1 . In terms of management, patients under opioid were offered naloxegol (20\%), psychology-based interventions (29\%), and referral to pain specialists to rationalize opioid use $(38 \%)$. The majority of the patients in the opioid group $(80 \%)$ were referred for tertiary opinion from other areas of the UK, as compared to the $60 \%$ of patients without chronic opioid use.

Conclusions Female patients with chronic functional GI disorders with associated extra-intestinal functional pain syndromes and psychological comorbidity are prescribed opioid therapy more frequently. These patients are at increased risk of developing eating difficulties requiring advanced nutritional support, which is known to be more complicated in opioid users. Access to multidisciplinary coordinated therapy including pain management and psychological therapy is required early in these patients to avoid these adverse outcomes.

\begin{tabular}{llll}
\multicolumn{1}{l}{ Abstract PWE-083 Table 1} & \\
\hline & $\begin{array}{l}\text { Opioids } \\
\text { (n.21) }\end{array}$ & $\begin{array}{l}\text { Non-opioids } \\
\text { (n.79) }\end{array}$ & $\mathrm{p}$ value \\
\hline Female/Male & $20 / 1(95 \%)$ & $66 / 13(84,6 \%)$ & $\mathrm{ns}$ \\
Age (mean \pm SD) & $36,5 \pm 16$ & $38,3 \pm 12$ & $\mathrm{~ns}$ \\
$\begin{array}{l}\text { Hypermobility Elhers- } \\
\text { Danlos }\end{array}$ & $11(52 \%)$ & $14(18 \%)$ & $\mathrm{p}<0.001$ \\
Depression & $4(38 \%)$ & $3(16,6 \%)$ & $\mathrm{p}<0.05$ \\
Artificial nutrition & $17(19 \%)$ & $32(5 \%)$ & $\mathrm{p}<0.05$ \\
\hline
\end{tabular}

\section{PWE-084 ANORECTAL PHYSIOLOGY PROFILES IN YOUNG ADULTS, MIDDLE-AGED ADULTS AND OLDER ADULTS WITH FAECAL INCONTINENCE}

Ismail Miah*. Guy's and St Thomas' Nhs Trust, Greater London, UK

\subsection{6/gutjnl-2019-BSGAbstracts.404}

Introduction Adult patients of all age report faecal incontinence (FI) symptoms but little has been done on comparing the anorectal physiology profiles in young adults (YA), middleaged adults (MAA) and older adults (OA). This study investigates the topic in question.

Method All patients selected for this study complained of FI and underwent anorectal physiology investigation that was performed by an experienced gastrointestinal physiologist between October 2010 and October 2013. Any patients with history of constipation or anorectal surgery were excluded. YA, MAA and OA patients were grouped into ages 1-0 years, >4-5 years and $>65$ years based on Erik Erikson's theory of adult development.

Patients' FI symptoms were sub-classified based on urge faecal incontinence (UFI) and passive faecal incontinence (PFI) symptoms. The anorectal physiology tests included manometry assessment of anal sphincter motor function [1], rectal balloon sensory to distension study [2] and anorectal mucosal electrical stimulation to assess for sensory deficit [3]. Each test were benchmarked against normal range [4]. Appropriate 3-group ANOVA and $\chi 2$ statistical tests were used to compare the anorectal physiology profiles in YA, MAA and OA.

Results The female: male ratio for YA, MAA and OA were respectively 59:10, 174:34 and 100:28.

YA showed significantly higher incidence in weak involuntary EAS contraction compared to MMA and OA patients $(\chi 2=6.0, p=0.0498)$. This was consistent with the clinical presentation as $74.2 \%$ YA patients only report UFI symptoms and UFI symptoms were less prevalence in MAA (57.1\%) and OA $(33.6 \%)(\mathrm{p}<0.00001)$.

In contrast to YA, PFI symptoms were more frequently reported by MMA and OA which can be explained by the combination of higher prevalence of hypotensive anal tone $(\chi 2=32.6, \quad \mathrm{p}<0.00001)$ and diminished sensory to rectal capacity volume $(\chi 2=82.0, \mathrm{p}<0.00001)$ observed in MMA and OA. This pathophysiology in the anorectal compliance found in MMA and OA would increase the risk of developing passive rectal overflow incontinence that patient's report as PFI symptoms. Furthermore, MAA and OA patients also have higher incidences of anal sensory deficit compared to YA $(\chi 2=10.4, p=0.0056)$ suggesting of unfelt anal seepage that is also perceived as PFI. 